\title{
On the influence of center-Lipschitz conditions in the convergence analysis of multi-point iterative methods
}

\author{
Ioannis K. Argyros ${ }^{1}$ and Santhosh George ${ }^{2}$
}

\begin{abstract}
The aim of this article is to extend the local as well as the semilocal convergence analysis of multi-point iterative methods using center Lipschitz conditions in combination with our idea, of the restricted convergence region. It turns out that this way a finer convergence analysis for these methods is obtained than in earlier works and without additional hypotheses. Numerical examples favoring our technique over earlier ones completes this article.
\end{abstract}

AMS Subject Classification: $65 \mathrm{~F} 08,37 \mathrm{~F} 50,65 \mathrm{~N} 12$.

Key Words: Multi-point iterative methods; Banach space; local-semilocal convergence analysis.

\section{Introduction}

Let $\mathcal{X}, \mathcal{Y}$ be Banach spaces and $\Omega \subset \mathcal{X}$ be a nonempty and open set. By $\mathcal{B}(\mathcal{X}, \mathcal{Y})$, we denote the space of bounded linear operators from $\mathcal{X}$ into $\mathcal{Y}$. Let also $U(w, d)$, be an open set centered at $w \in \mathcal{X}$ and of radius $d>0$ and $\bar{U}(w, d)$ be its closure.

Many problems from diverse disciplines such that Mathematics, Optimization, Mathematical Programming, Chemistry, Biology, Physics, Economics, Statistics, Engineering and other disciplines $[?, ?, ?, ?, ?, ?, ?, ?, ?, ?, ?, ?, ?, ?, ?, ?, ?, ?$, $?, ?, ?, ?, ?, ?, ?, ?, ?, ?, ?, ?, ?, ?]$, can be reduced to finding a solution $x^{*}$ of the equation

$$
\mathcal{H}(x)=0,
$$

where $\mathcal{H}: \Omega \longrightarrow \mathcal{Y}$ is a continuous operator. Since,a unique solution $x^{*}$ of equation (??) in a neighborhood of some initial data $x_{0}$ can be obtained only in special cases. Researchers construct iterative methods which generate a sequence converging to $x^{*}$.

The most widely used iterative method is Newton's defined for each $n=$ $0,1,2, \ldots$ by

$$
x_{0} \in \Omega, x_{n+1}=x_{n}-\mathcal{H}^{\prime}\left(x_{n}\right)^{-1} \mathcal{H}\left(x_{n}\right) .
$$

\footnotetext{
${ }^{1}$ Department of Mathematical Sciences, Cameron University, Lawton, OK 73505, USA, Email: iargyros@cameron.edu

${ }^{2}$ Department of Mathematical and Computational Sciences, National Institute of Technology Karnataka, India-575 025, Email:sgeorge@nitk.edu.in
} 
The order of convergence is an important concern when dealing with iterative methods. The computational cost increases in general especially when the convergence order increases.

That is why researchers and practitioners have developed iterative methods that on the one hand avoid the computation of derivatives and on the other hand achieve high order of convergence.

We consider the following multi-step iterative method defined for each $n=$ $0,1,2, \ldots$ by

$$
\begin{aligned}
u_{n} & =v_{n}^{(0)} \\
v_{n}^{(1)} & =v_{n}^{(0)}-\mathcal{H}^{\prime}\left(v_{n}^{(0)}\right)^{-1} \mathcal{H}\left(v_{n}^{(0)}\right) \\
v_{n}^{(2)} & =v_{n}^{(1)}-\mathcal{H}^{\prime}\left(v_{n}^{(0)}\right)^{-1} \mathcal{H}\left(v_{n}^{(1)}\right) \\
& \cdots \\
u_{n+1} & =v_{n}^{(k)}=v_{n}^{(k-1)}-\mathcal{H}^{\prime}\left(v_{n}^{(0)}\right)^{-1} \mathcal{H}\left(v_{n}^{(k-1)}\right) .
\end{aligned}
$$

The semi-local convergence of method (??) was given in [?]. It is well known that as the convergence order increases the convergence region decreases in general. To avoid this problem, we introduce a center-Lipschitz-type condition that helps us determine an at least as small region as before containing the iterates $\left\{u_{n}\right\}$. This way the resulting Lipschitz constants are at least as small. A tighter convergence analysis is obtained this way. The order of convergence was shown using Taylor expansions and conditions reaching up to the $k+1$ order derivative of $\mathcal{H}$, although these derivatives do not appear in this method. As an academic example: Let $\mathcal{X}=\mathcal{Y}=\mathbb{R}, \Omega=\left[-\frac{5}{2}, \frac{3}{2}\right]$. Define $\varphi$ on $\Omega$ by

$$
\varphi(x)=x^{3} \log x^{2}+x^{5}-x^{4}
$$

Then

$$
\begin{gathered}
\varphi^{\prime}(x)=3 x^{2} \log x^{2}+5 x^{4}-4 x^{3}+2 x^{2}, \\
\varphi^{\prime \prime}(x)=6 x \log x^{2}+20 x^{3}-12 x^{2}+10 x, \\
\varphi^{\prime \prime \prime}(x)=6 \log x^{2}+60 x^{2}=24 x+22 .
\end{gathered}
$$

Obviously $\varphi^{\prime \prime \prime}(x)$ is not bounded on $\Omega$. So, the convergence of methods (??) is not guaranteed by the analysis in $[?, ?, ?]$.

The rest of the article is organized as follows: Section 2 contains the conditions to be used in the semi-local convergence that follows in Section 3. Finally the numerical examples are given in the concluding Section 4.

\section{Local convergence}

Let $L_{0}>0, L>0$ and $L_{1} \geq 1$ be parameters. Define the scalar quadratic polynomial $p$ by

$$
p(t)=\left(2 L_{0}+L\right) L_{0} t^{2}-\left(4 L_{0}+4 L_{0} L_{1}+L\right) t+2 .
$$


The discriminant $D$ of $p$ is given by

$$
\begin{aligned}
D & =\left(4 L_{0}+4 L_{0} L_{1}+L\right)^{2}-8 L_{0}\left(2 L_{0}+L\right) \\
& =16\left(L_{0} L_{1}\right)^{2}+L^{2}+32 L_{0}^{2} L_{1}+8 L_{0} L_{1} L>0,
\end{aligned}
$$

so $p$ has roots $s_{1}$ and $s_{2}$ with $0<s_{1}<s_{2}$ by the Descarte's rule of signs. Define also parameters

$$
\gamma=\left(\frac{L}{2\left(1-L_{0} s_{1}\right)}+\frac{2 L_{0} L_{1}}{\left(1-L_{0} s_{1}\right)^{2}}\right) s_{1}
$$

and

$$
r_{A}=\frac{2}{2 L_{0}+L} .
$$

Notice that $\gamma \in(0,1]$, since $p\left(s_{1}\right)=0$. The local convergence analysis of method (??) uses the conditions (A):

(a1) $\mathcal{H}: \Omega \longrightarrow \mathcal{Y}$ is a differentiable operator in the sense of Fréchet and there exists $x_{*} \in \Omega$ such that $\mathcal{H}\left(x_{*}\right)=0$ and $\mathcal{H}^{\prime}\left(x_{*}\right)^{-1} \in \mathcal{L}(\mathcal{Y}, \mathcal{X})$.

(a2) There exists $L_{0}>0$ such that for each $x \in \Omega$

$$
\left\|\mathcal{H}^{\prime}\left(x_{*}\right)^{-1}\left(\mathcal{H}^{\prime}(x)-\mathcal{H}^{\prime}\left(x_{*}\right)\right)\right\| \leq L_{0}\left\|x-x_{*}\right\| .
$$

Set $\Omega_{0}=\Omega \cap B\left(x_{*}, \frac{1}{L_{0}}\right)$.

(a3) There exist $L=L\left(L_{0}\right)>0$ and $L_{1}=L_{1}\left(L_{0}\right) \geq 1$ such that for each $x, y \in \Omega_{0}$

$$
\left\|\mathcal{H}^{\prime}\left(x_{*}\right)^{-1}\left(\mathcal{H}^{\prime}(y)-\mathcal{H}^{\prime}(x)\right)\right\| \leq L\|x-y\|
$$

and

$$
\left\|\mathcal{H}^{\prime}\left(x_{*}\right)^{-1} \mathcal{H}^{\prime}(x)\right\| \leq L_{1}\left\|x-x_{*}\right\|
$$

(a4) $\bar{B}\left(x_{*}, s_{1}\right) \subset \Omega$.

(a5) There exists $s_{3} \geq s_{1}$ such that $s_{3}<\frac{2}{L_{0}}$. Set $\Omega_{1}=\Omega \cap \bar{B}\left(x_{*}, \frac{2}{L_{0}}\right)$.

Based on the preceding conditions and notations we can show a local convergence result for method (??).

THEOREM 2.1 Under the conditions (A), further assume that $u_{0} \in B\left(x_{*}, s_{1}\right)$ $\left\{x_{*}\right\}$. Then, $\lim _{n \rightarrow \infty} u_{n}=x_{*}$, and the following estimations hold

$$
\begin{gathered}
\left\|v_{n}^{1}-x_{*}\right\| \leq \frac{L\left\|v_{n}^{0}-x_{*}\right\|^{2}}{2\left(1-L_{0}\left\|v_{0}^{0}-x_{*}\right\|\right)}, \\
\left\|v_{n}^{i}-x_{*}\right\| \leq \gamma^{i}\left\|v_{n}^{0}-x_{*}\right\| \text { for each } i=2, \ldots, k
\end{gathered}
$$

and

$$
\left\|u_{n+1}-x_{*}\right\| \leq \gamma^{k+n}\left\|u_{0}-x_{*}\right\| .
$$

Moreover, the point $x_{*}$ is the unique solution of equation $\mathcal{H}(x)=0$ in the set $\Omega_{1}$ given in (a5). 
Proof. We use an induction based proof to show estimations (??)-(??). Let $x \in B\left(x_{*}, s_{1}\right)-\left\{x_{*}\right\}$. By (a1) and (a2), we obtain that

$$
\left\|\mathcal{H}^{\prime}\left(x_{*}\right)^{-1}\left(\mathcal{H}^{\prime}(x)-\mathcal{H}^{\prime}\left(x_{*}\right)\right)\right\| \leq L_{0}\left\|x-x_{*}\right\|<L_{0} s_{1}<1 .
$$

It follows from the Banach lemma on invertible operators [?] and (??) that $\mathcal{H}^{\prime}\left(x_{*}\right)^{-1} \in \mathcal{L}(\mathcal{Y}, \mathcal{X})$ and

$$
\left\|\mathcal{H}^{\prime}(x)^{-1} \mathcal{H}^{\prime}\left(x_{*}\right)\right\| \leq \frac{1}{1-L_{0}\left\|x-x_{*}\right\|} .
$$

Then, $x_{0}=v_{0}^{0}, v_{0}^{1}, \ldots, v_{0}^{k}$ are well defined by method (??) for $n=0$. We can write

$$
v_{0}^{1}-x_{*}=v_{0}^{0}-x_{*}-\mathcal{H}^{\prime}\left(v_{0}^{0}\right)^{-1} \mathcal{H}\left(v_{0}^{0}\right) .
$$

Then, by using (a1), (a3), (??) and (??), we get in turn that

$$
\begin{aligned}
\left\|v_{0}^{1}-x_{*}\right\| & =\left\|v_{0}^{0}-x_{*}-\mathcal{H}^{\prime}\left(v_{0}^{0}\right)^{-1} \mathcal{H}\left(v_{0}^{0}\right)\right\| \\
\leq & \left\|\mathcal{H}^{\prime}\left(v_{0}^{0}\right)^{-1} \mathcal{H}^{\prime}\left(x_{*}\right)\right\| \\
& \int_{0}^{1}\left\|\mathcal{H}^{\prime}\left(x_{*}\right)^{-1}\left[\mathcal{H}^{\prime}\left(x_{*}+\theta\left(v_{0}^{0}-x_{*}\right)\right)-\mathcal{H}^{\prime}\left(v_{0}^{0}\right)\right] d \theta\left(v_{0}^{0}-x_{*}\right)\right\| \\
& \leq \frac{L\left\|v_{0}^{0}-x_{*}\right\|^{2}}{2\left(1-L_{0}\left\|v_{0}^{0}-x_{*}\right\|\right)} \\
& <\frac{L s_{1}}{2\left(1-L_{0} s_{1}\right)}\left\|v_{0}^{0}-x_{*}\right\| \\
& <\left\|v_{0}^{0}-x_{*}\right\|<s_{1},
\end{aligned}
$$

which shows (??) for $n=0$ and $v_{0}^{1} \in B\left(x_{*}, s_{1}\right)$. Similarly by the second substep for $n=0, k=2$ we also get

$$
\begin{aligned}
v_{0}^{2}-x_{*}= & v_{0}^{1}-x_{*}-\mathcal{H}^{\prime}\left(v_{0}^{0}\right)^{-1} \mathcal{H}\left(v_{0}^{1}\right) \\
= & v_{0}^{1}-x_{*}-\mathcal{H}^{\prime}\left(v_{0}^{1}\right)^{-1} \mathcal{H}\left(v_{0}^{1}\right) \\
& +\mathcal{H}^{\prime}\left(v_{0}^{1}\right)^{-1}\left[\left(\mathcal{H}^{\prime}\left(v_{0}^{0}\right)-\mathcal{H}^{\prime}\left(x_{*}\right)\right)+\left(\mathcal{H}^{\prime}\left(x_{*}\right)-\mathcal{H}^{\prime}\left(v_{0}^{1}\right)\right)\right] \\
& \times \mathcal{H}^{\prime}\left(v_{0}^{0}\right)^{-1} \mathcal{H}\left(v_{0}^{1}\right)
\end{aligned}
$$

so by (a3), the definition of $s_{1}$ and (??) (for $x=v_{0}^{1}, v_{0}^{0}$ ), we get in turn that

$$
\begin{aligned}
\left\|v_{0}^{2}-x_{*}\right\| \leq & \frac{L\left\|v_{0}^{1}-x_{*}\right\|^{2}}{2\left(1-L_{0}\left\|v_{0}^{1}-x_{*}\right\|\right)} \\
& +\frac{L_{0}\left(\left\|v_{0}^{1}-x_{*}\right\|+\left\|v_{0}^{0}-x_{*}\right\|\right)}{\left(1-L_{0}\left\|v_{0}^{1}-x_{*}\right\|\right)\left(1-L_{0}\left\|v_{0}^{0}-x_{*}\right\|\right)} \\
\leq & \left(\frac{L}{2\left(1-L_{0} s_{1}\right)}+\frac{2 L_{0} L_{1}}{\left(1-:_{0} s_{1}\right)^{2}}\right) s_{1}\left\|v_{0}^{1}-x_{*}\right\| \\
\leq & \gamma s_{1}\left\|v_{0}^{1}-x_{*}\right\|<s_{1},
\end{aligned}
$$


which shows (??) for $n=0$ and $k=2$. Similarly, from

$$
\begin{aligned}
v_{0}^{i}-x_{*}= & v_{0}^{i-1}-x_{*}-\mathcal{H}^{\prime}\left(v_{0}^{0}\right)^{-1}\left(\mathcal{H}\left(v_{0}^{i-1}\right)\right) \\
= & v_{0}^{i-1}-x_{*}-\mathcal{H}^{\prime}\left(v_{0}^{i-1}\right) \mathcal{H}\left(v_{0}^{i-1}\right) \\
& +\mathcal{H}^{\prime}\left(v_{0}^{i-1}\right)^{-1}\left[\left(\mathcal{H}^{\prime}\left(v_{0}^{0}\right)-\mathcal{H}^{\prime}\left(x_{*}\right)\right)\right. \\
& \left.+\left(\mathcal{H}^{\prime}\left(x_{*}\right)-\mathcal{H}^{\prime}\left(v_{0}^{i-1}\right)\right)\right] \mathcal{H}^{\prime}\left(v_{0}^{0}\right)^{-1} \mathcal{H}\left(v_{0}^{i-1}\right)
\end{aligned}
$$

so

$$
\left\|v_{0}^{i}-x_{*}\right\| \leq \gamma\left\|v_{0}^{i-1}-x_{*}\right\| \leq \gamma^{i}\left\|v_{0}^{0}-x_{*}\right\|<s_{1},
$$

and

$$
\left\|x_{1}-x_{*}\right\| \leq \gamma^{k+1}\left\|x_{0}-x_{*}\right\|<s_{1},
$$

which show (??) and (??) for $n=1, i=2,3, \ldots, k$ and $v_{0}^{i}, x_{1} \in B\left(x_{*}, s_{1}\right)$. Similarly, by induction we have in turn (as in (??))

$$
\begin{aligned}
\left\|v_{j}^{1}-x_{*}\right\|= & \left\|v_{j}^{0}-x_{*}-\mathcal{H}^{\prime}\left(v_{0}^{j}\right)^{-1} \mathcal{H}\left(v_{j}^{0}\right)\right\| \\
\leq & \frac{L\left\|v_{j}^{0}-x_{*}\right\|^{2}}{2\left(1-L_{0}\left\|v_{j}^{0}-x_{*}\right\|\right)}<s_{1}, \\
\left\|v_{j}^{i}-x_{*}\right\|= & \| v_{j}^{i-1}-x_{*}-\mathcal{H}^{\prime}\left(v_{j}^{0}\right)^{-1} \mathcal{H}\left(v_{j}^{1}\right) \\
& +\mathcal{H}^{\prime}\left(v_{j}^{i-1}\right)^{-1}\left[\left(\mathcal{H}^{\prime}\left(v_{j}^{i-1}\right)-\mathcal{H}^{\prime}\left(x_{*}\right)\right)\right. \\
& \left.+\left(\mathcal{H}^{\prime}\left(x_{*}\right)-\mathcal{H}^{\prime}\left(v_{j}^{0}\right)\right)\right] \mathcal{H}^{\prime}\left(v_{j}^{0}\right)^{-1} \mathcal{H}\left(v_{j}^{i-1}\right) \|
\end{aligned}
$$

leading to (as in (??))

$$
\left\|v_{j}^{i}-x_{*}\right\| \leq \gamma^{i}\left\|v_{j}^{0}-x_{*}\right\|<s_{1}
$$

and (as in (??))

$$
\left\|u_{n+1}-x_{*}\right\|=\left\|v_{n}^{k}-x_{*}\right\| \leq \gamma^{k+n}\left\|u_{0}-x_{*}\right\|<s_{1},
$$

which completes the induction for (??)-(??) and also show that $u_{n+1} \in B\left(x_{*}, s_{1}\right)$. It also follows from (??) that $\lim _{n \rightarrow \infty} u_{n}=x_{*}$, since $\gamma \in[0,1)$. Let $x_{* *} \in \Omega_{1}$ with $\mathcal{H}\left(x_{* *}\right)=0$. It then follows from the definition of $\Omega_{1}$, (a2) and $T=$ $\int_{0}^{1} \mathcal{H}^{\prime}\left(x_{* *}+\tau\left(x_{*}-x_{* *}\right)\right) d \tau$ that

$$
\left\|\mathcal{H}^{\prime}\left(x_{*}\right)^{-1}\left(T-\mathcal{H}\left(x_{*}\right)\right)\right\| \leq \frac{L_{0}}{2} \int_{0}^{1}\left\|x_{*}-x_{* *}\right\| d \tau \leq \frac{L_{0}}{2} s_{3}<1,
$$

so $T^{-1} \in \mathcal{L}(\mathcal{Y}, \mathcal{X})$. Then, from the estimate

$$
0=\mathcal{H}\left(x_{*}\right)-\mathcal{H}\left(x_{* *}\right)=T\left(x_{*}-x_{* *}\right),
$$

we get $x_{*}=x_{* *}$. 
REMARK 2.2 (a) In view of (a2), we can write

$$
\begin{aligned}
\| \mathcal{H}^{\prime}\left(x_{*}\right)^{-1} \mathcal{H}^{\prime}(x) & =\left\|\mathcal{H}^{\prime}\left(x_{*}\right)^{-1}\left[\left(\mathcal{H}^{\prime}(x)-\mathcal{H}^{\prime}\left(x_{*}\right)\right)+\mathcal{H}^{\prime}\left(x_{*}\right)\right]\right\| \\
& \leq 1+\left\|\mathcal{H}^{\prime}\left(x_{*}\right)^{-1}\left(\mathcal{H}^{\prime}(x)-\mathcal{H}^{\prime}\left(x_{*}\right)\right)\right\| \\
& \leq 1+L_{0}\left\|x-x_{*}\right\|,
\end{aligned}
$$

so the second condition in (a3) can be dropped, and we choose $L_{1}=2$, since $\left\|x-x_{*}\right\| \leq s_{1}<\frac{1}{L_{0}}$.

(b) It follows from the definition of $s_{1}$ and $r_{A}$ that $s_{1}<r_{A}$. That is the radius of convergence $s_{1}$ cannot be larger than the radius of convergence $r_{A}$ of Newton's method obtained by us [?, ?, ?, ?, ?].

(c) The local convergence of method (??) was not studied in [?]. But if it was call; $\bar{s}_{1}$ the smallest positive solution of $\bar{p}(t)=0$, where

$$
\bar{p}(t)=\left(2 L_{0}+\bar{L}\right) L_{0} t^{2}-\left(4 L_{0}+4 L_{0} \bar{L}_{1}+\bar{L}\right) t+2,
$$

where $\bar{L}$ and $\bar{L}_{1}$ are the constants for the conditions in (a3) holding on $\Omega$. But, we have

$$
\begin{gathered}
L \leq \bar{L} \\
L_{1} \leq \bar{L}_{1}
\end{gathered}
$$

and

$$
\gamma \leq \bar{\gamma}
$$

since $\Omega_{0} \subset \Omega$. Hence, we have

$$
\bar{s}_{1} \leq s_{1} .
$$

Moreover, if strict inequality holds in (??) or (??), then, we have $\bar{s}_{1}<s_{1}$. Furthermore, by (??), our error bounds are more precise than the ones using $L_{0}, \bar{L}, \bar{L}_{1}$ and $\bar{\gamma}$. Hence, we have expanded the applicability of method (??) in the local convergence case.

In a similar way, we improve the semi-local convergence analysis of method (??) given in [?]. The work is given in the next section.

\section{Semi-local convergence}

We need the following auxiliary result on majorizing sequences for method (??).

LEMMA 3.1 Let $K_{0}>0, K>0$, and $r_{0}^{1}$ be parameters. Denote by $\delta$ the unique root in the interval $(0,1)$ of the polynomial $\varphi$ given by

$$
\varphi(t)=2 K_{0} t^{k+1}+K\left(t^{k}+t^{k-1}-2\right) .
$$

Define the sequence $\left\{q_{n}\right\}$ for each $n=0,1,2, \ldots$ and $i=1,2, \ldots, k-1$ by

$$
r_{0}^{0}=0, r_{n}^{0}=r_{n}, r_{n+1}^{1}=q_{n+1}+\frac{K\left(q_{n+1}-q_{n}+r_{n}^{k-1}-q_{n}\right)\left(q_{n+1}-r_{n}^{k-1}\right)}{1-2 K_{0} q_{n+1}}
$$


and

$$
r_{n}^{k}=q_{n+1}, r_{n}^{i+1}=r_{n}^{i}+\frac{K\left(r_{n}^{i}-q_{n}+r_{n}^{i-1}-q_{n}\right)\left(r_{n}^{i}-r_{n}^{i-1}\right)}{1-2 K_{0} q_{n}} .
$$

Moreover, suppose that

$$
0<\frac{K\left(q_{1}+r_{0}^{k-1}\right)}{1-2 K_{0} q_{1}} \leq \delta<1-2 K_{0} r_{0}^{1}
$$

Then, the sequence $\left\{q_{n}\right\}$ is increasing, bounded from above by $q_{* *}=\frac{r_{0}^{1}}{1-\alpha}$ and converges to its unique least upper bound $q_{*}$ satisfying $q_{1} \leq q_{*} \leq q_{* *}$,

$$
\begin{gathered}
r_{n}^{1}-r_{n}^{i-1} \leq \delta\left(r_{n}^{i-1}-r_{n}^{i-2}\right) \leq \delta^{k n+i-1} r_{0}^{1}, \\
r_{n+1}^{1}-r_{n}^{k} \leq \delta\left(r_{n}^{k}-r_{n}^{k-1}\right) \leq \delta^{k(n+1)} r_{0}^{1}
\end{gathered}
$$

and

$$
q_{n}=r_{n}^{0} \leq r_{n}^{1} \leq r_{n}^{2} \leq \ldots \leq r_{n}^{k-1} \leq r_{n}^{k}=q_{n+1} .
$$

Proof. Replace $t_{n}, s_{n}^{i}, L_{0}, L, \alpha$ in [?] by $q_{n}, r_{n}^{i}, K_{0}, K, \delta$.

Next, we present the semi-local convergence analysis of method (??).

THEOREM 3.2 Let $\mathcal{H}: \Omega \longrightarrow \mathcal{Y}$ be a continuously differentiable operator in the sense of Fréchet and $[., . ; \mathcal{H}]: \Omega \longrightarrow \mathcal{L}(\mathcal{X}, \mathcal{Y})$ be a divided difference of order one of $\mathcal{H}$. Suppose there exist $x_{0} \in \Omega$ and $K_{0}>0$ such that

$$
\begin{gathered}
\mathcal{H}^{\prime}\left(x_{0}\right)^{-1} \in \mathcal{L}(\mathcal{Y}, \mathcal{X}) . \\
\left\|\mathcal{H}^{\prime}\left(x_{0}\right)^{-1} \mathcal{H}\left(x_{0}\right)\right\| \leq r_{0}^{1}, \\
\left\|\mathcal{H}^{-1}\left(x_{0}\right)\left([x, y ; \mathcal{H}]-\mathcal{H}^{\prime}\left(x_{0}\right)\right)\right\| \leq K_{0}\left(\left\|x-x_{0}\right\|+\left\|y-x_{0}\right\|\right) .
\end{gathered}
$$

Set $\Omega_{2}=\Omega \cap B\left(x_{0}, \frac{1}{2 K_{0}}\right)$. Moreover, suppose that for each $x, y, z, w \in \Omega_{2}$

$$
\left\|\mathcal{H}^{\prime}\left(x_{0}\right)^{-1}([x, y ; \mathcal{H}]-[z, w ; \mathcal{H}])\right\| \leq K(\|x-z\|+\|y-w\|),
$$

and the hypotheses of Lemma ?? hold. Then, $\left\{u_{n}\right\} \in B\left(v_{0}, q_{*}\right), \lim _{n} \longrightarrow \infty u_{n}=$ $u_{*} \in \bar{B}\left(v_{0}, q_{*}\right), \mathcal{H}\left(u_{*}\right)=0$. and

$$
\left\|u_{*}-u_{n}\right\| \leq q_{*}-v_{n}
$$

Moreover, $u_{*}$ is the unique solution of equation $\mathcal{H}(x)=0$ in $\bar{B}\left(v_{0}, q_{*}\right)$.

Proof. Replace $x_{n}, y_{n}, s_{n}^{i}, t_{n}, \alpha, L_{0}, L$ in [?] by $u_{n}, v_{n}, r_{n}^{i}, q_{n}, \delta, K_{0}, K$.

REMARK 3.3 The condition

$$
\left\|\mathcal{H}^{\prime}\left(x_{0}\right)^{-1}([x, y ; \mathcal{H}]-[z, w ; \mathcal{H}])\right\| \leq L(\|x-z\|+\|y-w\|)
$$

for each $x, y, z, w \in \Omega$, some $L>0$ is used in [?] instead of (??). But we have

$$
K_{0}=L_{0}
$$




$$
K \leq L
$$

and

$$
\delta \leq \alpha,
$$

since $\Omega_{2} \subseteq \Omega$. Denote by $\bar{q}_{n}, \bar{r}_{n}^{i}$ the majorizing sequences used in [?] and defined as sequences $q_{n}, r_{n}^{i}$ but with $K_{0}=L_{0}$ and $K$ replaced by $L$. Then, we have by a simple induction argument, that

$$
\begin{aligned}
q_{n} & \leq \bar{q}_{n} \\
r_{n}^{i} & \leq \bar{r}_{n}^{i} \\
0 & \leq r_{n}^{i}-q_{n} \leq \bar{r}_{n}^{i}-\bar{q}_{n} \\
0 & \leq r_{n}^{i}-r_{n}^{i-1} \leq \bar{r}_{n}^{i}-\bar{r}_{n}^{i-1} \\
0 & \leq q_{n+1}-r_{n}^{k-1} \leq \bar{q}_{n}-\bar{r}_{n}^{k-1}
\end{aligned}
$$

and

$$
q_{*} \leq \bar{q}_{*} .
$$

Moreover, if $K<L$, then (??-(??) hold as strict inequalities. Let us consider the set $\Omega_{3}=\Omega \cap B\left(x_{1}, \frac{1}{2 K}-r_{0}^{1}\right)$ provided that $r_{0}^{1}<\frac{1}{2 K}$. Moreover, suppose for each $x, y, z, w \in \Omega_{3}$

$$
\left\|\mathcal{H}^{-1}\left(x_{0}\right)([x, y ; H]-[z, w ; H])\right\| \leq \lambda(\|x-z\|+\|y-w\|) .
$$

Notice that $\Omega_{3} \subseteq \Omega_{2}$, so $\lambda \leq K$. Then, $\Omega_{3}$, (??), $\lambda$ can replace $\Omega_{2}$, (??), and $K$ respectively in Theorem ??. Clearly, the corresponding to $\left\{t_{n}\right\}$ majorizing sequence call it $\left\{\bar{t}_{n}\right\}$ is even tighter than $\left\{t_{n}\right\}$. Hence, we have extended the applicability of method (??) in the semi-local convergence analysis too. These improvements are derived under the same conditions as in [?], since the computation of $L$ is included in the computation of $K$ as a special case. Examples where the new constants are smaller than the older ones can be found in the numerical section that follows and in [?, ?, ?, ?, ?].

\section{Numerical examples}

We present the following examples to test the convergence criteria. Define the divided difference by

$$
[x, y ; \mathcal{H}]=\int_{0}^{1} \mathcal{H}^{\prime}(\tau x+(1-\tau) y) d \tau .
$$

EXAMPLE 4.1 Let $\mathcal{X}=\mathcal{Y}=\mathbb{R}^{3}, \Omega=U(0,1), x^{*}=(0,0,0)^{T}$ and define $\mathcal{H}$ on $\Omega$ by

$$
\mathcal{H}(x)=\mathcal{H}\left(x_{1}, x_{2}, x_{3}\right)=\left(e^{x_{1}}-1, \frac{e-1}{2} x_{2}^{2}+x_{2}, x_{3}\right)^{T} .
$$


For the points $u=\left(u_{1}, u_{2}, u_{3}\right)^{T}$, the Fréchet derivative is given by

$$
\mathcal{H}^{\prime}(u)=\left(\begin{array}{ccc}
e^{u_{1}} & 0 & 0 \\
0 & (e-1) u_{2}+1 & 0 \\
0 & 0 & 1
\end{array}\right)
$$

Using the norm of the maximum of the rows and $\left(a_{3}\right)-\left(a_{4}\right)$ and since $\mathcal{H}^{\prime}\left(x^{*}\right)=$ $\operatorname{diag}(1,1,1)$, we can define parameters for method (??) by $L_{0}=e-1, L_{1}=L=$ $e^{\frac{1}{e-1}}, \bar{L}=\bar{L}_{1}=e$. Then, $s_{1}=0.0997$ The old radius is $\bar{s}_{1}=0.0727$.

EXAMPLE 4.2 Let $\mathcal{X}=\mathcal{Y}=\mathbb{R}, \Omega=\bar{U}\left(x_{0}, 1-\xi\right), x_{0}=1$ and $\xi \in\left[0, \frac{1}{2}\right)$. Define function $\mathcal{H}$ on $\Omega$ by

$$
\mathcal{H}(x)=x^{3}-\xi
$$

Then, we get by (??)-(??) and (??) that for

(i) $k=1, q_{1}=r_{0}^{1}=\frac{1}{3}(1-\xi), L_{0}=K_{0}=\frac{1}{2}(3-\xi), L=2-\xi$, and $K=$ $1+\frac{1}{2 K_{0}}$. Notice that $L_{0}<K<L$. The conditions of Lemma ?? are satisfied for $\xi \in I_{1}=[0.434523,0.5)$ but the earlier conditions in [?] are satisfied for $\xi \in I_{2}=[0.464523,0.5)$, and $I_{2} \subset I_{1}$. Moreover, if $\lambda=\frac{1}{3(3-\xi)}\left(-2 \xi^{2}+5 \xi+6\right)$ then conditions of Lemma ?? are satisfied for $\xi \in I_{3}=[0.3720452,0.5)$.

(ii)For $k=2$, the conditions of Lemma ?? are satisfied for $\xi \in I_{1}=[0.6161045,0.7)$ but the earlier conditions in [?] are satisfied for $\xi \in I_{2}=[0.6266523,0.7)$, and $I_{2} \subset I_{1}$. Moreover, if $\lambda=\frac{1}{3(3-\xi)}\left(-2 \xi^{2}+5 \xi+6\right)$ then conditions of Lemma ?? are satisfied for $\xi \in I_{3}=[0.5966523,0.7)$.

\section{Conclusion}

Our idea of the convergence region in connection to the center Lipschitz condition were utilized to provide a local as well as a semilocal convergence analysis of method (??). Due to the fact that we locate a region at least as small as in earlier works [?] containing the iterates, the new Lipschitz parameters are also at least as small. This technique leads to a finer convergence analysis (see also Remark ??, Remark ?? and the numerical examples). The novelty of the paper not only lies in the introduction of the new idea but also obtained using special cases of Lipschitz parameters appearing in [?]. Hence, no additional work to [?] is needed to arrive at these developments. This idea can be used to extend the applicability of other iterative methods appearing $[?, ?, ?, ?, ?, ?, ?, ?, ?, ?, ?, ?, ?, ?, ?, ?, ?, ?, ?, ?, ?, ?, ?, ?, ?, ?, ?, ?, ?, ?]$ along the same lines.

\section{References}

[1] S. Amat, I. K. Argyros, S. Busquier, and M. A. Herńandez-Veŕon. On two high-order families of frozen newton-type methods. Numerical Linear Algebra with Applications, 25(1), 2018. 
[2] S. Amat, C. Berḿudez, M.A. Herńandez-Veŕon, and E. Martinez. On an efficient k-step iterative method for nonlinear equations. Journal of Computational and Applied Mathematics, 302:258-271, 2016.

[3] S. Amat, S. Busquier, C. Berḿudez, and S. Plaza, On two families of high order newton type methods. Applied Mathematics Letters, 25(12):22092217, 2012.

[4] S. Amat, S. Busquier, and S. Plaza, Review of some iterative root-finding methods from a dynamical point of view. Scientia, 10(3):35, 2004.

[5] I.K. Argyros, Computational theory of iterative methods, volume 15. Elsevier, 2007.

[6] I. K. Argyros, On the semilocal convergence of a fast two-step newton method. Revista Colombiana de Matematicas, 42(1):15-24, 2008.

[7] I. K. Argyros, S. George, N. Thapa, Mathematical Modeling For The Solution Of Equations And Systems Of Equations With Applications, Volume-I, Nova Publishes, NY, 2018.

[8] I. K. Argyros, S. George, N. Thapa, Mathematical Modeling For The Solution Of Equations And Systems Of Equations With Applications, VolumeII, Nova Publishes, NY, 2018.

[9] I. K. Argyros, A. Cordero, A. A. Magreñán, and J. R. Torregrosa, Thirddegree anomalies of traubs method. Journal of Computational and Applied Mathematics, 309:511-521, 2017.

[10] I. K. Argyros and S. Hilout, Weaker conditions for the convergence of newtons method. Journal of Complexity, 28(3):364-387, 2012.

[11] I. K. Argyros and S. Hilout, Computational methods in nonlinear analysis: efficient algorithms, fixed point theory and applications. World Scientific, 2013.

[12] I. K. Argyros, A. A. Magreñán, L. Orcos, and J.A Sicilia, Local convergence of a relaxed two-step newton like method with applications. Journal of Mathematical Chemistry, 55(7):1427- 1442, 2017.

[13] I. K.Argyros, A. A. Magréñan, A contemporary study of iterative methods, Elsevier (Academic Press), New York, 2018.

[14] I. K.Argyros, A. A. Magréñan, Iterative methods and their dynamics with applications, CRC Press, New York, USA, 2017.

[15] A.Cordero, L. Guasp, and J. R. Torregrosa, Choosing the most stable members of kous family of iterative methods. Journal of Computational and Applied Mathematics, 2017. 
[16] A. Cordero and J. R. Torregrosa, Low-complexity root-finding iteration functions with no derivatives of any order of convergence. Journal of Computational and Applied Mathematics, 275:502-515, 2015.

[17] A. Cordero, J. R. Torregrosa, and P. Vindel, Study of the dynamics of thirdorder iterative methods on quadratic polynomials. International Journal of Computer Mathematics, 89(13-14):1826-1836, 2012.

[18] J.A. Ezquerro and M.A. Herńandez-Veŕon, How to improve the domain of starting points for steffensen's method. Studies in Applied Mathematics, 132(4):354-380, 2014.

[19] J. A. Ezquerro and M. A. Herńandez-Veŕon, Majorizing sequences for nonlinear fredholm hammerstein integral equations. Studies in Applied Mathematics.

[20] J. A. Ezquerro, M. Grau-Śanchez, M. A. Herńandez-Veŕon, and M. Noguera, A family of iterative methods that uses divided differences of first and second orders. Numerical algorithms, 70(3):571-589, 2015.

[21] J. A. Ezquerro, M.A .Herńandez-Veŕon, and A. I. Velasco, An analysis of the semilocal convergence for secant-like methods. Applied Mathematics and Computation, 266:883-892, 2015.

[22] M.A. Herńandez-Veŕon, E. Martínez, and C. Teruel, Semilocal convergence of a k-step iterative process and its application for solving a special kind of conservative problems. Numerical Algorithms, 76(2):309-331, 2017.

[23] L.V. Kantlorovich and G.P. Akilov, Functional analysis pergamon press, 1982.

[24] M. Moccari and T. Lofti, Using majorizing sequences for the semi-local convergence of a high order and multi-point method along with stability analysis, Intern.J. Non. Sci. and Numer. Stimulation,

[25] A. A. Magreñán, A.Cordero, J. M. Gutiérrez, and J. R. Torregrosa, Real qualitative behavior of a fourth-order family of iterative methods by using the convergence plane. Mathematics and Computers in Simulation, 105:4961, 2014 .

[26] A. A. Magreñán and I. K .Argyros, Improved convergence analysis for Newton-like methods. Numerical Algorithms, 71(4):811-826, 2016.

[27] A. A. Magreñán and I. K. Argyros, Two-step newton methods. Journal of Complexity, 30(4):533-553, 2014.

[28] F.A. Potra and V. Pták, Nondiscrete induction and iterative processes, volume 103. Pitman Advanced Publishing Program, 1984. 
[29] Rheinboldt, W.C., An adaptive continuation process for solving systems of nonlinear equations, Polish Academy of Science, Banach Ctr. Publ. 3 (1978), no. 1, 129-142.

[30] H.Ren and I. K. Argyros, On the convergence of kingwerner-type methods of order free of derivatives. Applied Mathematics and Computation, 256:148-159, 2015. R volume 1. Cambridge University

[31] W.T. Shaw, Complex analysis with Mathematical Press, 2006.

[32] J. F. Traub, Iterative methods for the solution of equations. American Mathematical Soc., 1982. 